\title{
Numerical Solution of Volterra Integral Equations with Weakly Singular Kernels which May Have a Boundary Singularity*
}

\section{Kolk and A. Pedas}

Institute of Mathematics, University of Tartu

J. Liivi 2, 50409 Tartu, Estonia

E-mail: marek.kolk@ut.ee

E-mail(corresp.): arvet.pedas@ut.ee

Received October 30, 2008; published online February 25, 2009

\begin{abstract}
We propose a piecewise polynomial collocation method for solving linear Volterra integral equations of the second kind with kernels which, in addition to a weak diagonal singularity, may have a weak boundary singularity. Global convergence estimates are derived and a collection of numerical results is given.
\end{abstract}

Key words: Volterra integral equation, weakly singular kernel, boundary singularity, collocation method.

\section{Introduction}

Let $C^{k}(\Omega)$ be the set of all $k$ times continuously differentiable functions on $\Omega$, $C^{0}(\Omega)=C(\Omega)$. Let $b \in \mathbb{R}=(-\infty, \infty), b>0$,

$$
D_{b}=\{(x, y): 0 \leq x \leq b, 0<y<x\}, \quad \bar{D}_{b}=\{(x, y): 0 \leq y \leq x \leq b\} .
$$

In many practical applications (see, for example, $[3,5]$ ) there arise integral equations of the form

$$
u(x)=\int_{0}^{x} K(x, y) u(y) d y+f(x), \quad 0 \leq x \leq b,
$$

with $f \in C^{m}[0, b], K(x, y)=g(x, y)(x-y)^{-\nu}, 0<\nu<1, g \in C^{m}\left(\bar{D}_{b}\right)$, $m \in \mathbb{N}=\{1,2, \ldots\}$. The solution $u(x)$ to (1.1) is typically non-smooth at $x=0$ where its derivatives become unbounded (see, for example, $[3,4,5,9]$ ). In collocation methods the singular behaviour of the solution $u(x)$ can be taken into account by using polynomial splines on special graded grids

$$
\Delta_{N}^{r}=\left\{x_{0}, \ldots, x_{N}: 0=x_{0}<\ldots<x_{N}=b\right\}
$$

\footnotetext{
* This work was supported by EstSF grant No. 7353
} 
with the nodes

$$
x_{i}=b(i / N)^{r}, \quad i=0, \ldots, N, \quad N \in \mathbb{N}, \quad r \in \mathbb{R}, \quad r \geq 1 .
$$

The parameter $r$ characterizes the degree of non-uniformity of the grid $\Delta_{N}^{r}$ : if $r>1$, then the nodes $x_{0}, \ldots, x_{N}$ of the grid $\Delta_{N}^{r}$ are more densely clustered near the left endpoint of the interval $[0, b]$ where $u(x)$ may be singular. By using a collocation method based on the grid $\Delta_{N}^{r}$ and piecewise polynomials of degree at most $m-1$ one can reach a convergence of order $\mathcal{O}\left(N^{-m}\right)$ for $r \geq m /(1-\nu)$, see $[3,4,5]$. However, although the piecewise polynomial collocation method on $\Delta_{N}^{r}$ turns out to be stable for solving weakly singular integral equations (see [8]), the realization of this method in case of strongly graded grids $\Delta_{N}^{r}$ by large values of $r$ may lead to unstable behaviour of numerical results.

To avoid problems associated with the use of strongly graded grids the following approach for solving (1.1) can be used: first we perform in (1.1) a change of variables so that the singularities of the derivatives of the solution will be milder or disappear and after that we solve the transformed equation by a collocation method on a mildly graded or uniform grid. We refer to [13] for details (see also $[2,7,12]$ ). Note that in $[10,15]$ similar ideas for solving Fredholm integral equations have been used (see also $[6,11,16]$ ).

In the present paper we extend the domain of applicability of this approach. To this aim, we examine a more complicated situation for equation (1.1) where the kernel $K(x, y)$, in addition to a diagonal singularity (a singularity as $y \rightarrow x$ ), may have a boundary singularity (a singularity as $y \rightarrow 0$ ). Actually, we assume that the kernel $K(x, y)$ has the form

$$
K(x, y)=g(x, y)(x-y)^{-\nu} y^{-\lambda}, \quad(x, y) \in D_{b}, \quad 0<\nu<1, \quad 0 \leq \lambda<1
$$

where $g \in C^{m}\left(\bar{D}_{b}\right), m \in\{0\} \cup \mathbb{N}$. The set of kernels satisfying (1.3) will be denoted by $W^{m, \nu, \lambda}\left(D_{b}\right)$.

Throughout the paper $c$ denotes a positive constant which may have different values by different occurrences.

\section{Regularity of the Solution}

For given $m \in \mathbb{N}$ and $0<\theta<1$ let $C^{m, \theta}(0, b]$ be the set of functions $u \in$ $C[0, b] \cap C^{m}(0, b]$ such that

$$
\left|u^{(j)}(x)\right| \leq c x^{1-\theta-j}, \quad 0<x \leq b, \quad j=1, \ldots, m .
$$

It follows from [14] that the regularity of the solution to (1.1) can be characterized by the following result.

Lemma 1. Assume that $K \in W^{m, \nu, \lambda}\left(D_{b}\right)$ and $f \in C^{m, \nu+\lambda}(0, b]$ where $m \in \mathbb{N}$, $0<\nu<1,0 \leq \lambda<1, \nu+\lambda<1$. Then equation (1.1) has a unique solution $u \in C^{m, \nu+\lambda}(0, b]$. 


\section{Smoothing Transformation}

For given $\varrho \in[1, \infty)$ denote

$$
\varphi(s)=b^{1-\varrho} s^{\varrho}, \quad 0 \leq s \leq b .
$$

Clearly, $\varphi \in C[0, b], \varphi(0)=0, \varphi(b)=b$ and $\varphi^{\prime}(s)>0$ for $0<s \leq b$. Thus, $\varphi$ maps $[0, b]$ onto $[0, b]$ and has a continuous inverse $\varphi^{-1}:[0, b] \rightarrow[0, b]$,

$$
\varphi^{-1}(x)=b^{(\varrho-1) / \varrho} x^{1 / \varrho}, \quad 0 \leq x \leq b .
$$

Note that $\varphi(s) \equiv s$ for $\varrho=1$. We are interested in a transformation (3.1) with $\varrho>1$ since it possesses a smoothing property for $u(\varphi(s))$ with singularities of $u^{\prime}(x), \ldots, u^{(m)}(x)$ at $x=0$ (see Lemma 2).

Lemma 2. Let $u \in C^{m, \theta}(0, b], m \in \mathbb{N}, 0<\theta<1$, and let $\varphi$ be the transformation (3.1). Furthermore, let

$$
u_{\varphi}(s)=u(\varphi(s)), \quad 0 \leq s \leq b .
$$

Then $u_{\varphi} \in C[0, b] \cap C^{m}(0, b]$ and

$$
\left|u_{\varphi}^{(j)}(s)\right| \leq c s^{\varrho(1-\theta)-j}, \quad 0<s \leq b, \quad j=1, \ldots, m .
$$

Proof. The smoothness claim is clear. Further, for the derivatives of the composite function $u_{\varphi}=u \circ \varphi$, we have the Faà di Bruno's representation

$$
u_{\varphi}^{(j)}(s)=\sum \frac{j !}{n_{1} ! \ldots n_{j} !} u^{(n)}(\varphi(s))\left(\frac{\varphi^{\prime}(s)}{1 !}\right)^{n_{1}} \ldots\left(\frac{\varphi^{(j)}(s)}{j !}\right)^{n_{j}}
$$

where $0<s \leq b, n=n_{1}+\ldots+n_{j}$ and the sum is taken over all $n_{1}, \ldots, n_{j} \in$ $\{0\} \cup \mathbb{N}$ for which $n_{1}+2 n_{2}+\ldots+j n_{j}=j, j=1, \ldots, m$. It follows from (2.1), (3.1), $n=n_{1}+\ldots+n_{j}$ and $n_{1}+2 n_{2}+\ldots+j n_{j}=j$ that

$$
\left|u^{(n)}(\varphi(s))\left(\varphi^{\prime}(s)\right)^{n_{1}} \ldots\left(\varphi^{(j)}(s)\right)^{n_{j}}\right| \leq c s^{\varrho(1-\theta)-j}, \quad 0<s \leq b .
$$

This together with (3.3) yields (3.2).

Remark 1. Instead of (3.1) other transformations are possible. We refer to [13] for a general discussion in this connection.

\section{Numerical Method}

Using (3.1) we introduce in (1.1) the change of variables $y=\varphi(s), x=\varphi(t)$, $s, t \in[0, b]$. We obtain an integral equation of the form

$$
u_{\varphi}(t)=\int_{0}^{t} K_{\varphi}(t, s) u_{\varphi}(s) d s+f_{\varphi}(t), \quad 0 \leq t \leq b,
$$

where

$$
f_{\varphi}(t)=f(\varphi(t)), \quad K_{\varphi}(t, s)=K(\varphi(t), \varphi(s)) \varphi^{\prime}(s)
$$


are given functions and $u_{\varphi}(t)=u(\varphi(t))$ is a function which we have to find.

For given integers $m, N \in \mathbb{N}$ let

$$
\begin{aligned}
& S_{m-1}^{(-1)}\left(\Delta_{N}^{r}\right)=\left\{v_{N}:\left.v_{N}\right|_{\left[x_{j-1}, x_{j}\right]} \in \pi_{m-1}, j=1, \ldots, N\right\} \\
& S_{m-1}^{(0)}\left(\Delta_{N}^{r}\right)=\left\{v_{N} \in C[0, b]:\left.v_{N}\right|_{\left[x_{j-1}, x_{j}\right]} \in \pi_{m-1}, j=1, \ldots, N\right\}
\end{aligned}
$$

be the underlying spline spaces of piecewise polynomial functions on the grid $\Delta_{N}^{r}$ with the nodes (1.2). Here $\left.v_{N}\right|_{\left[x_{j-1}, x_{j}\right]}(j=1, \ldots, N)$ is the restriction of $v_{N}(t), t \in[0, b]$, to the subinterval $\left[x_{j-1}, x_{j}\right] \subset[0, b]$ and $\pi_{m-1}$ denotes the set of polynomials of degree not exceeding $m-1$. Note that the elements of $S_{m-1}^{(-1)}\left(\Delta_{N}^{r}\right)$ may have jump discontinuities at the interior knots $x_{1}, \ldots, x_{N-1}$ of the grid $\Delta_{N}^{r}$. In every subinterval $\left[x_{j-1}, x_{j}\right](j=1, \ldots, N)$ we introduce $m \in \mathbb{N}$ interpolation (collocation) points

$$
x_{j l}=x_{j-1}+\eta_{l}\left(x_{j}-x_{j-1}\right), \quad l=1, \ldots, m ; j=1, \ldots, N,
$$

where $\eta_{1}, \ldots, \eta_{m}$ are some fixed (collocation) parameters such that

$$
0 \leq \eta_{1}<\ldots<\eta_{m} \leq 1 .
$$

We find an approximation $v_{N}=v_{N, m, r, \varphi}$ to $u_{\varphi}$, the solution of equation (4.1) (under the conditions of Theorem 1 below the equations (1.1) and (4.1) are uniquely solvable), by collocation method from the following conditions:

$$
\begin{aligned}
& v_{N} \in S_{m-1}^{(-1)}\left(\Delta_{N}^{r}\right), \quad N, m \in \mathbb{N}, r \geq 1 \\
& v_{N}\left(x_{j l}\right)=\int_{0}^{x_{j l}} K_{\varphi}\left(x_{j l}, s\right) v_{N}(s) d s+f_{\varphi}\left(x_{j l}\right), \quad l=1, \ldots, m ; j=1, \ldots, N,
\end{aligned}
$$

with $x_{j l}, l=1, \ldots, m ; j=1, \ldots, N$, given by formula $(4.2)$.

Having determined the approximation $v_{N}$ for $u_{\varphi}$, we determine an approximation $u_{N}=u_{N, m, r, \varphi}$ for $u$, the solution of equation (1.1), setting

$$
u_{N}(x)=v_{N}\left(\varphi^{-1}(x)\right), \quad 0 \leq x \leq b .
$$

Remark 2. The choice of nodes (4.2) with $\eta_{1}=0, \eta_{m}=1$ in (4.5) actually implies that the resulting collocation approximation $v_{N}$ belongs to the smoother spline space $S_{m-1}^{(0)}\left(\Delta_{N}^{r}\right)$ than it is stated by the condition (4.4).

Remark 3. The settings (4.4), (4.5) form a linear system of algebraic equations whose exact form is determined by the choice of a basis in $S_{m-1}^{(-1)}\left(\Delta_{N}^{r}\right)$. We refer to [13] for a convenient choice of it.

\section{Convergence Results}

Let $X$ and $Y$ be Banach spaces. By $\mathcal{L}(X, Y)$ we denote the Banach space of all linear continuous operators $A: X \rightarrow Y$ with the norm

$$
\|A\|_{\mathcal{L}(X, Y)}=\sup \left\{\|A z\|_{Y}: z \in X,\|z\|_{X} \leq 1\right\} .
$$

By $C[a, b]$ we denote the Banach space of continuous functions $z$ on $[a, b]$ with the usual norm $\|z\|=\max \{|z(t)|: t \in[a, b]\}$. 
Theorem 1. Let $f \in C[0, b]$ and $K \in W^{0, \nu, \lambda}\left(D_{b}\right), 0<\nu<1,0 \leq \lambda<1-\nu$. Furthermore, assume that $\varphi$ is the transformation (3.1) and the interpolation nodes (4.2) with grid points (1.2) and parameters (4.3) are used. Then equation (1.1) has a unique solution $u \in C[0, b]$, the settings (4.4)-(4.6) determine for sufficiently large $N$ a unique approximation $u_{N}$ for $u$ and

$$
\left\|u_{N}-u\right\|_{\infty} \rightarrow 0 \quad \text { as } \quad N \rightarrow \infty
$$

where $\left\|u_{N}-u\right\|_{\infty}=\sup _{0 \leq x \leq b}\left|u_{N}(x)-u(x)\right|$.

Proof. We write (4.1) in the form $u_{\varphi}=T_{\varphi} u_{\varphi}+f_{\varphi}$ where $T_{\varphi}$ is defined by formula

$$
\left(T_{\varphi} z\right)(t)=\int_{0}^{t} K_{\varphi}(t, s) z(s) d s, \quad 0 \leq t \leq b .
$$

It follows from (1.3) and (3.1) that $K_{\varphi}(t, s)$ is continuous in $D_{b}$ and

$$
\left|K_{\varphi}(t, s)\right| \leq c(t-s)^{-\nu} s^{-\lambda}, \quad(t, s) \in D_{b} .
$$

Since $\nu+\lambda<1, T_{\varphi}$ is compact as an operator from $L^{\infty}(0, b)$ into $C[0, b]$, see [14]. This together with $f_{\varphi} \in C[0, b]$ yields that equation $u_{\varphi}=T_{\varphi} u_{\varphi}+f_{\varphi}$ (equation (4.1)) has a unique solution $u_{\varphi} \in C[0, b]$. In particular, (1.1) has a unique solution $u \in C[0, b]$.

Further, conditions $(4.4),(4.5)$ have the operator equation representation

$$
v_{N}=P_{N} T_{\varphi} v_{N}+P_{N} f_{\varphi}
$$

where $P_{N}$ is an operator which assigns to every continuous function $z \in C[0, b]$ its piecewise polynomial function $P_{N} z \in S_{m-1}^{(-1)}\left(\Delta_{N}^{r}\right)$ such that $\left(P_{N} z\right)\left(x_{j l}\right)=$ $z\left(x_{j l}\right), l=1, \ldots, m ; j=1, \ldots, N$. It follows from [17] that the norms of $P_{N} \in \mathcal{L}\left(C[0, b], L^{\infty}(0, b)\right)$ are bounded by a constant $c$ which is independent of $N$,

$$
\left\|P_{N}\right\|_{\mathcal{L}\left(C[0, b], L^{\infty}(0, b)\right)} \leq c
$$

and

$$
\left\|z-P_{N} z\right\|_{\infty} \rightarrow 0 \quad \text { as } \quad N \rightarrow \infty \text { for every } z \in C[0, b] .
$$

Using a standard argumentation (cf. [13, 15, 17]) we obtain that equation (5.2) has for sufficiently large values of $N$, say $N \geq N_{0}$, a unique solution $v_{N} \in S_{m-1}^{(-1)}\left(\Delta_{N}^{r}\right)$ and

$$
\left\|v_{N}-u_{\varphi}\right\|_{\infty} \leq c\left\|u_{\varphi}-P_{N} u_{\varphi}\right\|_{\infty}, \quad N \geq N_{0}
$$

Here $u_{\varphi}$ is the solution of equation (4.1) and $c$ is a positive constant not depending on $N$. Since $u_{\varphi} \in C[0, b]$, we get from (5.4) and (5.5) that $\left\|v_{N}-u_{\varphi}\right\|_{\infty} \rightarrow 0$ as $N \rightarrow \infty$. This together with

$$
\left\|u_{N}-u\right\|_{\infty}=\left\|v_{N}-u_{\varphi}\right\|_{\infty}
$$

yields (5.1).

Next we establish a global convergence result for method (4.4)-(4.6). 
Theorem 2. Let the following conditions be fulfilled:

1. $K \in W^{m, \nu, \lambda}\left(D_{b}\right), f \in C^{m, \nu+\lambda}(0, b], m \in \mathbb{N}, 0<\nu<1,0 \leq \lambda<1-\nu$;

2. $\varphi$ is the transformation (3.1);

3. the interpolation nodes (4.2) with grid points (1.2) and parameters (4.3) are used.

Then the settings (4.4)-(4.6) determine for $N \geq N_{0}$ a unique approximation $u_{N}$ to $u$, the solution to (1.1), and

$$
\left\|u_{N}-u\right\|_{\infty} \leq c \begin{cases}N^{-r \varrho(1-\nu-\lambda)} & \text { for } 1 \leq r<\frac{m}{\varrho(1-\nu-\lambda)} \\ N^{-m} & \text { for } r \geq \frac{m}{\varrho(1-\nu-\lambda)}, r \geq 1\end{cases}
$$

where $c$ is a positive constant not depending on $N$.

Proof. On the basis of Lemmas 1 and 2 we find that $u_{\varphi} \in C[0, b] \cap C^{m}(0, b]$ and for every $s \in(0, b]$ and $j=1, \ldots, m$,

$$
\left|u_{\varphi}^{(j)}(s)\right| \leq c \begin{cases}1 & \text { if } j \leq \varrho(1-\nu-\lambda) \\ s^{\varrho(1-\nu-\lambda)-j} & \text { if } j>\varrho(1-\nu-\lambda)\end{cases}
$$

For a spline $w_{N} \in S_{m-1}^{(-1)}\left(\Delta_{N}^{r}\right)$ denote $w_{N, j}=\left.w_{N}\right|_{\left[x_{j-1}, x_{j}\right]}, j=1, \ldots, N$. Due to (5.3) we get the estimate

$$
\begin{aligned}
\left\|u_{\varphi}-P_{N} u_{\varphi}\right\|_{\infty} & =\left\|u_{\varphi}-w_{N}-P_{N}\left(u_{\varphi}-w_{N}\right)\right\|_{\infty} \\
& \leq c \max _{j=1, \ldots, N} \max _{x_{j-1} \leq x \leq x_{j}}\left|u_{\varphi}(x)-w_{N, j}(x)\right|,
\end{aligned}
$$

with a positive constant $c$ which is independent of $N$. We fix $w_{N, j}$ as a Taylor polynomial for $u_{\varphi}(x)$ at $x=x_{j}$ :

$$
w_{N, j}(x)=\sum_{k=0}^{m-1} \frac{u_{\varphi}^{(k)}\left(x_{j}\right)}{k !}\left(x-x_{j}\right)^{k}, \quad x_{j-1} \leq x \leq x_{j} .
$$

The integral form of the reminder term of the $(m-1)$ th order Taylor approximation of $u_{\varphi}(x)$ at $x=x_{j}$ and the estimate (5.8) gives us for all $x \in\left[x_{j-1}, x_{j}\right]$ $(j=1, \ldots, N)$ the inequality

$$
\left|u_{\varphi}(x)-w_{N, j}(x)\right| \leq c \int_{x}^{x_{j}}(s-x)^{m-1}\left\{\begin{array}{lll}
1 & \text { if } & m \leq \varrho(1-\nu-\lambda) \\
s^{\varrho(1-\nu-\lambda)-m} & \text { if } & m>\varrho(1-\nu-\lambda)
\end{array}\right\} d s .
$$

Due to $(1.2)$,

$$
x_{j}-x_{j-1} \leq b r N^{-1}, \quad j=1, \ldots, N .
$$

If $m \leq \varrho(1-\nu-\lambda)$, then we obtain from (5.10) and (5.11) that

$$
\left|u_{\varphi}(x)-w_{N, j}(x)\right| \leq c N^{-m}, \quad x_{j-1} \leq x \leq x_{j}, \quad j=1, \ldots, N,
$$


where $c$ is a positive constant not depending on $N$.

In the case $m>\varrho(1-\nu-\lambda)$ we have

$$
\begin{aligned}
& \max _{0 \leq x \leq x_{1}} \int_{x}^{x_{1}}(s-x)^{m-1} s^{\varrho(1-\nu-\lambda)-m} d s \leq \max _{0 \leq x \leq x_{1}} \int_{x}^{x_{1}}(s-x)^{\varrho(1-\nu-\lambda)-1} d s \\
& \quad \leq c_{1}\left\{\begin{array}{lr}
N^{-\varrho r(1-\nu-\lambda)} & \text { for } \quad 1 \leq r<\frac{m}{\varrho(1-\nu-\lambda)}, \\
N^{-m} & \text { for } \quad r \geq \frac{m}{\varrho(1-\nu-\lambda)}, r \geq 1,
\end{array}\right. \\
& \max _{j=2, \ldots, N} \max _{x_{j-1} \leq x \leq x_{j}} \int_{x}^{x_{j}}(s-x)^{m-1} s^{\varrho(1-\nu-\lambda)-m} d s \\
& \quad \leq \max _{j=2, \ldots, N x_{j-1} \leq x \leq x_{j}} x^{\varrho(1-\nu-\lambda)-m \int_{x}^{x_{j}}(s-x)^{m-1} d s} \\
& \quad \leq c_{2} \begin{cases}N^{-\varrho r(1-\nu-\lambda)} & \text { for } \quad 1 \leq r<\frac{m}{\varrho(1-\nu-\lambda)}, \\
N^{-m} & \text { for } \quad r \geq \frac{m}{\varrho(1-\nu-\lambda)}, r \geq 1,\end{cases}
\end{aligned}
$$

where $c_{1}$ and $c_{2}$ are some positive constants not depending on $N$. It follows from $(5.9),(5.10)$ and $(5.12)-(5.14)$ that

$$
\left\|u_{\varphi}-P_{N} u_{\varphi}\right\|_{\infty} \leq c \begin{cases}N^{-r \varrho(1-\nu-\lambda)} & \text { for } 1 \leq r<\frac{m}{\varrho(1-\nu-\lambda)}, \\ N^{-m} & \text { for } r \geq \frac{m}{\varrho(1-\nu-\lambda)}, r \geq 1\end{cases}
$$

with a positive constant $c$ which is independent of $N$. This together with (5.5) and (5.6) yields (5.7).

Remark 4. It follows from Theorem 2 that the accuracy $\left\|u_{N}-u\right\|_{\infty} \leq c N^{-m}$ can be achieved on a mildly graded or uniform grid. As an example, if we assume that $\nu=2 / 5, \lambda=1 / 5, m=3$ (the case of piecewise quadratic polynomials), $\varrho \geq 15 / 2$, the maximal convergence order $\left\|u_{N}-u\right\|_{\infty} \leq c N^{-3}$ is available for $r \geq 1$. In particular, the uniform grid with nodes (1.2), $r=1$, may be used.

Remark 5. In addition to Theorem 2, assuming some additional smoothness of $f$ and $g$ (see (1.3)) and choosing more carefully the collocation parameters (4.3), the superconvergence of $v_{N}$ at the collocation points (4.2) can be established, cf. $[1,3,4,5,13,17]$. More precisely, let $K \in W^{m+1, \nu, \lambda}\left(D_{b}\right), f \in C^{m+1, \nu+\lambda}(0, b]$, $m \in \mathbb{N}, 0<\nu<1,0 \leq \lambda<1-\nu$, and let the interpolation nodes (4.2) be generated by the grid points $(1.2)$ and by the node points $\eta_{1}, \ldots, \eta_{m}$ of a quadrature approximation

$$
\int_{0}^{1} z(s) d s \approx \sum_{l=1}^{m} w_{l} z\left(\eta_{l}\right), \quad 0 \leq \eta_{1}<\ldots<\eta_{m} \leq 1
$$

which, with appropriate weights $\left\{w_{l}\right\}$, is exact for all polynomials of degree $m$. 
Then it turns out that for sufficiently large $N$,

$$
\begin{aligned}
& \max _{\substack{l=1, \ldots, m ; \\
j=1, \ldots, N}}\left|u_{N}\left(\varphi\left(x_{j l}\right)\right)-u\left(\varphi\left(x_{j l}\right)\right)\right|=\max _{\substack{l=1, \ldots, m ; \\
j=1, \ldots, N}}\left|v_{N}\left(x_{j l}\right)-u_{\varphi}\left(x_{j l}\right)\right| \\
& \leq c\left\{\begin{array}{lll}
N^{-2 \varrho}(1-\nu-\lambda) & \text { for } 1 \leq r<\frac{m+1-\nu}{2 \varrho(1-\nu-\lambda)}, \\
N^{-m-(1-\nu)} & \text { for } \quad r \geq \frac{m+1-\nu}{2 \varrho(1-\nu-\lambda)}, \quad r \geq 1 .
\end{array}\right.
\end{aligned}
$$

We will investigate this question in a forthcoming paper where a more general class of integral equations with diagonal and boundary singularities will be discussed.

\section{Numerical Example}

Let us consider the following equation:

$$
u(x)=\int_{0}^{x}(x-y)^{-\nu} y^{-\lambda} u(y) d y+f(x), \quad 0 \leq x \leq 1,
$$

where $0<\nu<1, \quad 0 \leq \lambda<1, \nu+\lambda<1$. The forcing function $f$ is selected so that $u(x)=x^{1-\nu-\lambda}$ is the exact solution to (6.1). Actually, this is a problem of the form (1.1), (1.3) where $b=1, g(x, y) \equiv 1, K(x, y)=(x-y)^{-\nu} y^{-\lambda}$,

$$
\begin{aligned}
f(x) & =x^{1-\nu-\lambda}-x^{2(1-\nu-\lambda)} \frac{\Gamma(1-\nu) \Gamma(2(1-\lambda)-\nu)}{\Gamma(3-2(\nu+\lambda))}, \quad 0 \leq x \leq 1, \\
\Gamma(t) & =\int_{0}^{\infty} e^{-s} s^{t-1} d s, \quad t>0 .
\end{aligned}
$$

It is easy to check that in this case $K \in W^{m, \nu, \lambda}\left(D_{1}\right)$ and $f \in C^{m, \nu+\lambda}(0,1]$ for arbitrary $m \in \mathbb{N}$.

Equation (6.1) was solved numerically by method (4.4)-(4.6) for $\nu=2 / 5$, $\lambda=1 / 5, m=3, \eta_{1}=(5-\sqrt{15}) / 10, \eta_{2}=1 / 2, \eta_{3}=(5+\sqrt{15}) / 10$. Here $\eta_{1}, \eta_{2}, \eta_{3}$ are the node points of the Gauss-Legendre quadrature rule (5.15) by $m=3$. This formula is exact for all polynomials of degree not exceeding $2 m-1=5$.

In Tables 1 and 2 some results for different values of the parameters $N, \varrho$ and $r$ are presented. The quantities $\varepsilon_{N}^{(\varrho, r)}$ in Table 1 are approximate values of the norm $\left\|u_{N}-u\right\|_{\infty}$, calculated as follows:

$$
\varepsilon_{N}^{(\varrho, r)}=\max _{\substack{l=0, \ldots, 10 \\ j=1, \ldots, N}}\left|u_{N}\left(\left(\tau_{j l}^{(r)}\right)^{\varrho}\right)-u\left(\left(\tau_{j l}^{(r)}\right)^{\varrho}\right)\right|
$$

where $\tau_{j l}^{(r)}=x_{j-1}+l\left(x_{j}-x_{j-1}\right) / 10, \quad l=0, \ldots, 10 ; \quad j=1, \ldots, N$, with the grid points $x_{j}$, defined by formula (1.2) for $b=1$.

Table 2 shows the dependence of

$$
\gamma_{N}^{(\varrho, r)}=\max _{\substack{l=1, \ldots, m ; \\ j=1, \ldots, N}}\left|u_{N}\left(\varphi\left(x_{j l}\right)\right)-u\left(\varphi\left(x_{j l}\right)\right)\right|=\max _{\substack{l=1, \ldots, m ; \\ j=1, \ldots, N}}\left|v_{N}\left(x_{j l}\right)-u_{\varphi}\left(x_{j l}\right)\right|
$$


on the parameters $N, \varrho$ and $r$ (see (5.16)). The ratios $\delta_{N}^{(\varrho, r)}=\varepsilon_{N / 2}^{(\varrho, r)} / \varepsilon_{N}^{(\varrho, r)}$, $\widetilde{\delta}_{N}^{(\varrho, r)}=\gamma_{N / 2}^{(\varrho, r)} / \gamma_{N}^{(\varrho, r)}$, characterizing the observed convergence rate, are also presented. From Theorem 2 it follows that for sufficiently large $N$,

$$
\varepsilon_{N}^{(\varrho, r)} \approx\left\|u_{N}-u\right\|_{\infty} \leq c \begin{cases}N^{-2 \varrho r / 5} & \text { if } 1 \leq \varrho r<15 / 2, \\ N^{-3} & \text { if } \varrho r \geq 15 / 2 .\end{cases}
$$

Table 1. $\left(m=3, \nu=\frac{2}{5}, \lambda=\frac{1}{5}, \eta_{1}=\frac{5-\sqrt{15}}{10}, \eta_{2}=\frac{1}{2}, \eta_{3}=\frac{5+\sqrt{15}}{10}\right)$

\begin{tabular}{cccccc}
\hline$N$ & $\varepsilon_{N}^{(1,1)}$ & $\varepsilon_{N}^{(3,1)}$ & $\varepsilon_{N}^{(7 / 2,3 / 2)}$ & $\varepsilon_{N}^{(15 / 2,1)}$ & $\varepsilon_{N}^{(15 / 4,2)}$ \\
& $\delta_{N}^{(1,1)}$ & $\delta_{N}^{(3,1)}$ & $\delta_{N}^{(7 / 2,3 / 2)}$ & $\delta_{N}^{(15 / 2,1)}$ & $\delta_{N}^{(15 / 4,2)}$ \\
\hline \multirow{2}{*}{32} & $7.7 \mathrm{E}-2$ & $3.1 \mathrm{E}-4$ & $1.7 \mathrm{E}-5$ & $1.8 \mathrm{E}-6$ & $9.2 \mathrm{E}-7$ \\
& 1.35 & 2.30 & 4.29 & 8.64 & 8.75 \\
64 & $5.8 \mathrm{E}-2$ & $1.3 \mathrm{E}-4$ & $3.9 \mathrm{E}-6$ & $2.1 \mathrm{E}-7$ & $1.1 \mathrm{E}-7$ \\
& 1.34 & 2.30 & 4.29 & 8.47 & 8.05 \\
128 & $4.3 \mathrm{E}-2$ & $5.8 \mathrm{E}-5$ & $9.1 \mathrm{E}-7$ & $2.6 \mathrm{E}-8$ & $1.4 \mathrm{E}-8$ \\
& 1.33 & 2.30 & 4.29 & 8.32 & 8.00 \\
256 & $3.2 \mathrm{E}-2$ & $2.5 \mathrm{E}-5$ & $2.1 \mathrm{E}-7$ & $3.1 \mathrm{E}-9$ & $1.8 \mathrm{E}-9$ \\
& 1.33 & 2.30 & 4.29 & 8.22 & 8.00 \\
512 & $2.4 \mathrm{E}-2$ & $1.1 \mathrm{E}-5$ & $4.9 \mathrm{E}-8$ & $3.9 \mathrm{E}-10$ & $2.2 \mathrm{E}-10$ \\
& 1.33 & 2.30 & 4.29 & 8.14 & 8.00 \\
\hline & 1.33 & 2.30 & 4.29 & 8.00 & 8.00 \\
\hline
\end{tabular}

Due to $(6.2)$, the ratio $\delta_{N}^{(\varrho, r)}$ ought to be approximately

$$
(N / 2)^{-2 \varrho r / 5} / N^{-2 \varrho r / 5}=2^{2 \varrho r / 5} \text { for } 1 \leq \varrho r<\frac{15}{2}
$$

and 8 for $\varrho r \geq 15 / 2$. In particular, $\delta_{N}^{(1,1)}, \delta_{N}^{(3,1)}, \delta_{N}^{\left(\frac{7}{2}, \frac{3}{2}\right)}, \delta_{N}^{\left(\frac{15}{2}, 1\right)}$ and $\delta_{N}^{\left(\frac{15}{4}, 2\right)}$ ought to be approximately $1.33,2.30,4.29,8.00$ and 8.00 , respectively. These values of $\delta_{N}^{(\varrho, r)}$ are given in the last row of Table 1 .

Table 2. $\left(m=3, \nu=\frac{2}{5}, \lambda=\frac{1}{5}, \eta_{1}=\frac{5-\sqrt{15}}{10}, \eta_{2}=\frac{1}{2}, \eta_{3}=\frac{5+\sqrt{15}}{10}\right)$

\begin{tabular}{cccccc}
\hline \multirow{2}{*}{$N$} & $\gamma_{N}^{(1,1)}$ & $\gamma_{N}^{(3,1)}$ & $\gamma_{N}^{(4,1)}$ & $\gamma_{N}^{(3,3 / 2)}$ & $\gamma_{N}^{(4,2)}$ \\
& $\widetilde{\delta}_{N}^{(1,1)}$ & $\widetilde{\delta}_{N}^{(3,1)}$ & $\widetilde{\delta}_{N}^{(4,1)}$ & $\widetilde{\delta}_{N}^{(3,3 / 2)}$ & $\widetilde{\delta}_{N}^{(4,2)}$ \\
\hline 32 & $1.4 \mathrm{E}-2$ & $3.5 \mathrm{E}-7$ & $3.8 \mathrm{E}-8$ & $1.7 \mathrm{E}-8$ & $8.0 \mathrm{E}-8$ \\
& 2.30 & 5.34 & 9.23 & 11.74 & 11.91 \\
64 & $6.3 \mathrm{E}-3$ & $6.5 \mathrm{E}-8$ & $4.2 \mathrm{E}-9$ & $1.4 \mathrm{E}-9$ & $6.8 \mathrm{E}-9$ \\
& 2.30 & 5.28 & 9.20 & 11.95 & 11.81 \\
128 & $2.7 \mathrm{E}-3$ & $1.2 \mathrm{E}-8$ & $4.5 \mathrm{E}-10$ & $1.2 \mathrm{E}-10$ & $5.7 \mathrm{E}-10$ \\
& 2.30 & 5.28 & 9.19 & 12.04 & 11.92 \\
256 & $1.2 \mathrm{E}-3$ & $2.4 \mathrm{E}-9$ & $4.9 \mathrm{E}-11$ & $9.6 \mathrm{E}-12$ & $4.7 \mathrm{E}-11$ \\
& 2.30 & 5.28 & 9.19 & 12.08 & 12.01 \\
512 & $5.2 \mathrm{E}-4$ & $4.5 \mathrm{E}-10$ & $5.4 \mathrm{E}-12$ & $7.9 \mathrm{E}-13$ & $3.9 \mathrm{E}-12$ \\
& 2.30 & 5.28 & 9.19 & 12.10 & 12.06 \\
\hline & 1.74 & 5.28 & 9.19 & 12.13 & 12.13 \\
\hline
\end{tabular}


In a similar way we obtain from $(5.16)$ that $\widetilde{\delta}_{N}^{(1,1)}, \widetilde{\delta}_{N}^{(3,1)} \widetilde{\delta}_{N}^{(4,1)}, \widetilde{\delta}_{N}^{\left(3, \frac{3}{2}\right)}$ and $\widetilde{\delta}_{N}^{(4,2)}$ ought to be approximately $1.74,5.28,9.19,12.13$ and 12.13 , respectively. These values of $\widetilde{\delta}_{N}^{(\varrho, r)}$ are given in the last row of Table 2.

As we can see from Tables 1 and 2, the numerical results are in good agreement with the theoretical estimates. In Table 2 only the decrease of $\gamma_{N}^{(1,1)}$ is faster than it is indicated by theoretical estimates: the predicted value for $\widetilde{\delta}_{N}^{(1,1)}$ is equal to 1.74 , but the current experiment gave for $\widetilde{\delta}_{N}^{(1,1)}$ a stable value 2.30. This phenomenon notifies that the local order of convergence of proposed algorithms needs further theoretical and numerical study.

\section{References}

[1] K. E. Atkinson. The Numerical Solution of Integral Equations of the Second Kind. Cambridge University Press, Cambridge, 1997.

[2] P. Baratella and A. P. Orsi. A new approach to the numerical solution of weakly singular Volterra integral equations. J. Comput. Appl. Math., 163:401-418, 2004.

[3] H. Brunner. Collocation Methods for Volterra Integral and Related Functional Equations, Cambridge Monographs on Applied and Computational Mathematics, 15. Cambridge University Press, 2004.

[4] H. Brunner, A. Pedas and G. Vainikko. The piecewise polynomial collocation method for nonlinear weakly singular Volterra equations. Math. Comput., 68:1079-1095, 1999.

[5] H. Brunner and P. J. van der Houwen. The Numerical Solution of Volterra Equations, CWI Monographs, 3. Amsterdam, North Holland, 1986.

[6] Y. Cao, M. Huang, L. Liu and Y. Xu. Hybrid collocation methods for Fredholm integral equations with weakly singular kernels. Appl. Numer. Math, 57:549-561, 2007.

[7] T. Diogo, S. McKee and T. Tang. Collocation methods for second-kind Volterra integral equations with weakly singular kernels. Proc. Roy. Soc. Edinburgh, 124:199-210, 1994.

[8] R. Kangro and I. Kangro. On the stability of piecewise polynomial collocation methods for solving weakly singular integral equations of the second kind. Math. Model. Anal., 13:29-36, 2008. (doi:10.3846/1392-6292.2008.13.29-36)

[9] R. K. Miller and A. Feldstein. Smoothness of solutions of Volterra integral equations with weakly singular kernels. SIAM J. Math. Anal., 2:242-258, 1971.

[10] G. Monegato and L. Scuderi. High order methods for weakly singular integral equations with nonsmooth input functions. Math. Comput., 67:1493-1515, 1998.

[11] R. Pallav and A. Pedas. Quadratic spline collocation method for weakly singular integral equations and corresponding eigenvalue problem. Math. Model. Anal., 7(2):285-296, 2002.

[12] A. Pedas and G. Vainikko. Numerical solution of weakly singular Volterra equations with change of variables. Proc. Estonian Acad. Sci. Phys. Math, 53(2):99106,2004 . 
[13] A. Pedas and G. Vainikko. Smoothing transformation and piecewise polynomial collocation for weakly singular Volterra integral equations. Computing, 73:271293, 2004.

[14] A. Pedas and G. Vainikko. Integral equations with diagonal and boundary singularities of the kernel. ZAA, 25(4):487-516, 2006.

[15] A. Pedas and G. Vainikko. Smoothing transformation and piecewise polynomial projection methods for weakly singular Fredholm integral equations. Commun. Pure Appl. Anal., 5:395-413, 2006.

[16] E. Vainikko and G. Vainikko. A spline product quasi-interpolation method for weakly singular Fredholm integral equations. SIAM J. Numer. Anal., 46:1799 $1820,2008$.

[17] G. Vainikko. Multidimensional Weakly Singular Integral Equations. SpringerVerlag, Berlin, 1993. 